\title{
Postharvest Storage Procedures and Oxidative Stress
}

\author{
Peter M.A. Toivonen ${ }^{1}$ \\ Agriculture and Agri-Food Canada, Pacific Agri-Food Research Centre, Summerland, British Columbia, \\ Canada VOH 1 ZO
}

Additional index words. reactive oxygen species, fruits, vegetables, storage disorders, browning, antioxidant systems

Oxidative stress in fruits and vegetables can be detected either directly (as accumulations in reactive oxygen species, increases in lipid peroxidation products, enhanced membrane leakage, or accumulation of brown pigments) or indirectly (as changes in antioxidant components or antioxidant enzyme systems). How all of these measures of oxidative stress relate to each other and how they are associated with the development of postharvest disorders have not been previously discussed in a generalized overview. While there have been great strides in understanding the interrelation of stress perception by the plant tissue and the attempt by the tissues to cope with that stress in the plant eco-physiology literature, postharvest physiology literature of fruits and vegetables has not advanced as far. The intent of this discussion is to bridge the understanding from eco-physiology of plants to improve perspective and interpretation of observations which currently exists in the postharvest physiology literature.

\section{CELLULAR LOCALIZATION OF OXYGEN RADICAL PRODUCTION}

Oxidative stress can be induced at various subcellular sites and initiated by an increased production of reactive oxygen species as a result of numerous processes (Fig. 1). In general, there are three sites for generation of reactive oxygen species: 1) the apoplastic region (cell wall, apoplastic space, the external surface of the plasma membrane), 2) the cytoplasm, and 3) cellular organelles. The three organelles which are most understood, in regards to reactive oxygen species generation, are chloroplasts, mitochondria, and peroxisomes/glyoxysomes. Two sites which have not been studied significantly are the nucleus and the vacuole (Mittler, 2002). Chloroplasts may not be an important site for reactive oxygen species generation in regards to postharvest situations, except perhaps in the harvesting and pre-precooling stage and also in situations where product is exposed to sufficient light to drive photosynthetic electron transport (e.g., perhaps a highly illuminated retail display).

Reactive oxygen species such as the superoxide anion $\left(\mathrm{O}_{2}^{-}\right)$, hydrogen peroxide $\left(\mathrm{H}_{2} \mathrm{O}_{2}\right)$, nitric oxide $\left(\mathrm{NO}^{*}\right)$, and peroxynitrite (ONOO') are relatively stable; however if they are allowed to accumulate, they can be precursors for the production of a more toxic reactive oxygen species, the hydroxyl radical $\left(\mathrm{OH}^{*}\right)$ (Stahl and Sies, 2002), which is linked with lipid peroxidation and membrane degradation (Scandalios, 1993). $\mathrm{H}_{2} \mathrm{O}_{2}$ and $\mathrm{NO}^{\circ}$ are considered as potential wound signaling agents both in the intra- and inter-cellular regions, largely because these two radicals are relatively stable (i.e., have long half-lives) and they can readily permeate membrane systems (Neill et al., 2002). Most importantly, as a result of their relative stability, their concentration can be regulated, i.e., if a stress episode passes the levels of these signal molecules will immediately decline, thus terminating the stress signal. There has been an indication that $\mathrm{O}_{2}{ }^{-}$can also act as a wound signal messenger in some situations (Overmyer et al., 2003).

An emerging concept in plant stress physiology is that oxidative reactions, stress, and

Received for publication 2 Feb. 2004. Accepted for publication 2 Feb. 2004. The author would like to thank Sabina Stan and Colin Kay for their helpful comments and feedback.

${ }^{1}$ E-mail toivonenp@agr.gc.ca. oxidatively-mediated response to stress are a normal course of affairs in plant tissues and the systems of signaling and response to any biotic and abiotic stress, no matter how mild or severe, involves reactive oxygen species (Mahalingam and Fedoroff, 2003; Mittler, 2002; Neill et al., 2002; Overmyer et al., 2003). In fact, there are low levels of superoxide anion and hydrogen peroxide found in nonstressed plant tissues (Mittler, 2002). As internal production of reactive oxygen species overwhelm or exceed the cell system's ability to cope, cellular or subcellular damage ensues (Lesham and Kuiper, 1996; Overmyer et al., 2003). This concept is at least two dimensional in complexity and these dimensions can best be understood by viewing Table 1 and Fig. 2 . Table 1 is a summary of the dynamics of stress response and the onset of stress and stress response, which is divided into three stages. The initial response is an alarm reaction to stress and leads to the production of reactive oxygen species. As a consequence of this, a number of modifications in plant cellular metabolism are initiated, including enhancement of antioxidant protection systems and gene regulation to effect metabolic changes that may ameliorate the stress response (Stage II). However, if the stress continues or the level of stress is extreme, the system can no longer cope, and at that point subcellular and cellular damage can occur (Stage III). Looking at the system as depicted in Fig.2, it is clear that oxidative regulation is a dynamic balancing process between systems that produce reactive oxygen species and systems which work to scavenge them and ameliorate the stress in an attempt to reduce reactive species production. When a severe stress is perceived, the existing production of reaction oxygen species is significantly enhanced and an inducible source of reactive oxygen species (NADPH oxidases in the plasmalemma) is initiated. The plant cell responds with a significant accentuation of gene expression to produce protein systems involved with protection. A good example of this is the production of heat shock proteins (Lesham and Kuiper, 1996). The result of all these features is that the plant cell has a complex system, which

Fig. 1. Reactive oxygen species produced by plant cells and the sites at which they are generated. Diagram is based on information from del Río (2002), Mettler (2002), and Mahalingam and Federoff (2003). $\mathrm{H}_{2} \mathrm{O}_{2}=$ hydrogen peroxide, $\mathrm{O}_{2}{ }^{-}=$superoxide anion, $\mathrm{PSII}=$ photosystem II, $\mathrm{PSI}=$ photosystem I, TCA $=$ tricarboxylic acid cycle, cyt. $\mathrm{c}=$ cytochrome $\mathrm{c}, \mathrm{ONOO}^{-}=$peroxynitrite radical, $\mathrm{NO}^{\circ}=$ nitric oxide, $\mathrm{NOS}=$ nitric oxide synthase, $\mathrm{XOD}=$ xanthine oxidase, and L-Arg $=$ L-arginine.

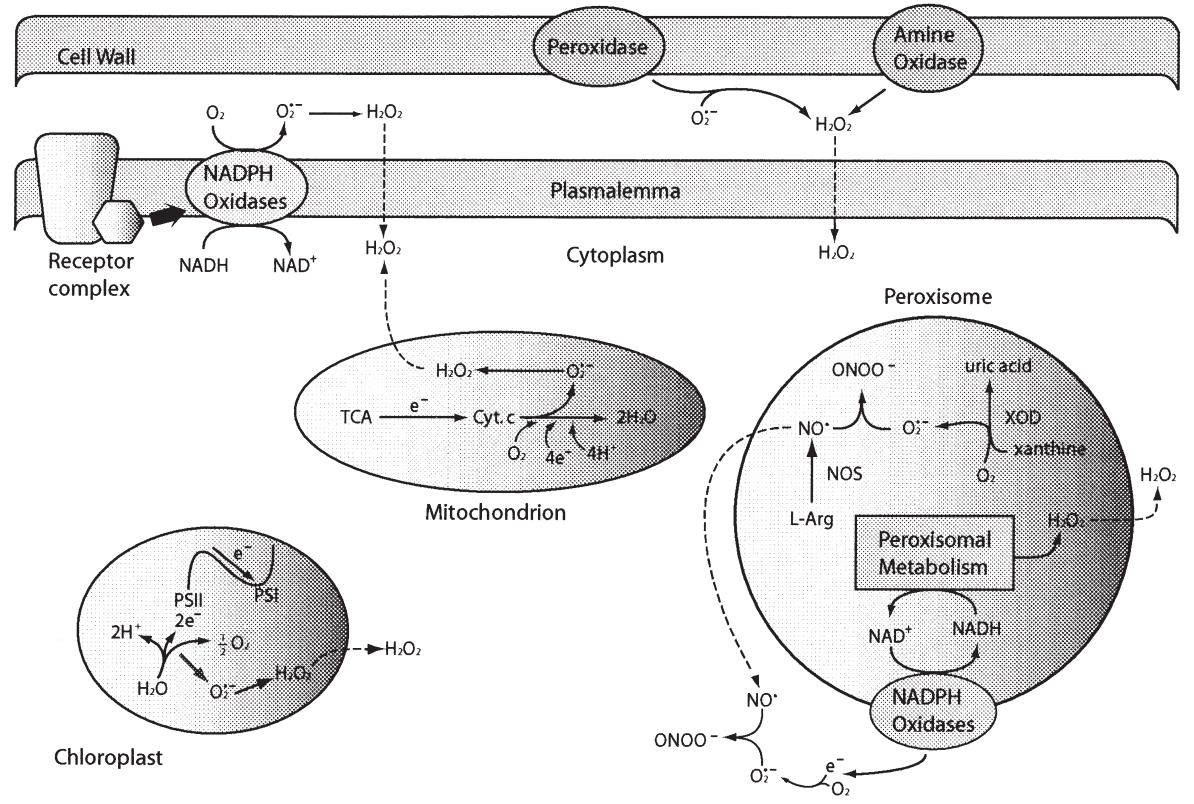


Table1. Stages of plant tissue response to an oxidative stressor and examples of indicators of the progression in stages from initial response, attempts to cope, and through to a point of cellular collapse. This table was developed from an original concept by Lesham and Kuiper (1996).

\begin{tabular}{|c|c|c|}
\hline Stage & Physiological state & Example measures indicative of stage \\
\hline $\mathrm{I}$ & Alarm reaction & Oxidative burst, increased production of superoxide anions, increased production of hydrogen peroxide. \\
\hline II & Resistance or acclimatization & $\begin{array}{l}\text { Modified gene expression, enhanced antioxidant enzyme activities, enhanced nonenzymatic antioxidants, production } \\
\text { of heat shock proteins. }\end{array}$ \\
\hline III & Collapse or exhaustion & $\begin{array}{l}\text { DNA/RNA damage, enhanced lipid peroxidation, loss of antioxidant enzyme activities, loss of nonenzymatic } \\
\text { antioxidants, increased membrane leakage, visible injury symptoms. }\end{array}$ \\
\hline
\end{tabular}

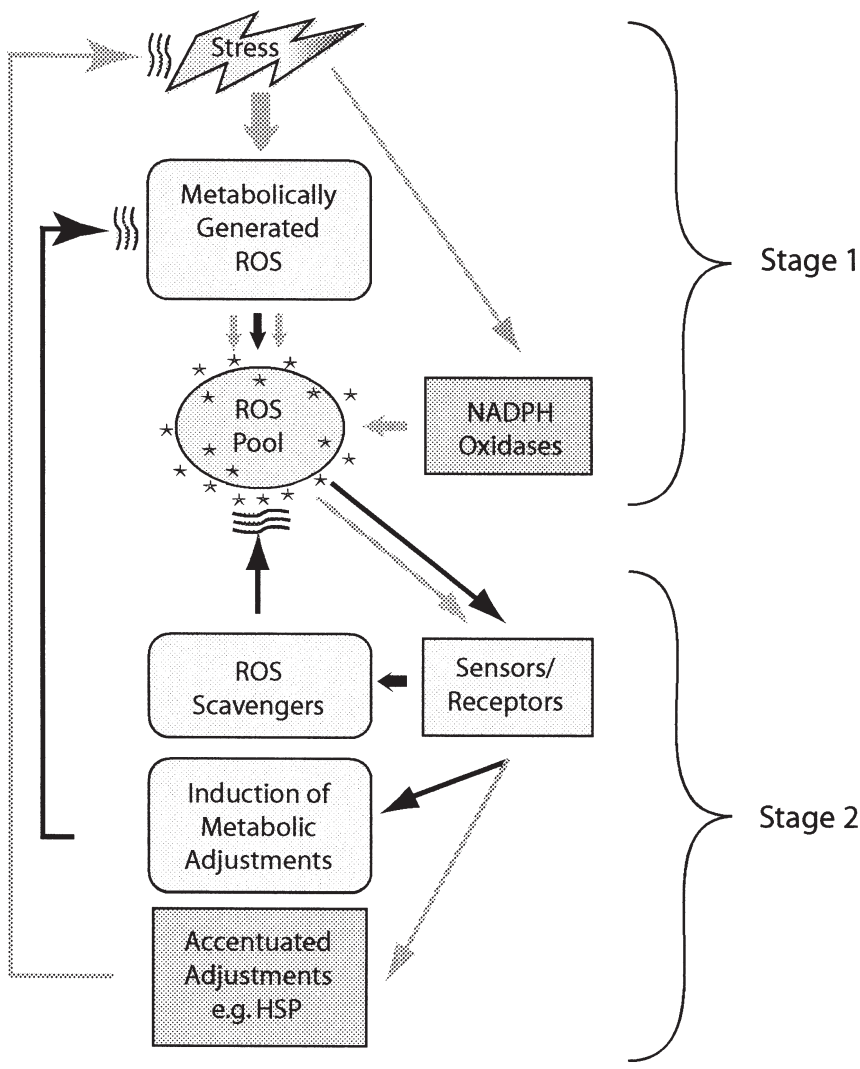

Fig. 2. Model depicting the association between stress response and the antioxidant protection components within a plant cell. The centre portion of the model represents the normal functioning systems, which includes a certain level of ROS (reactive oxygen species) production, allowing a small accumulation of ROS within the cells. This accumulation is monitored and regulated via various sensors and receptors in the cell. In a nonstress or low stress situation, regulation of ROS levels is achieved with minor modulations of ROS scavenging systems and through slight adjustments of metabolic/coping systems such that the production of ROS can also be modulated. In a moderate to severe stress situation additional pressure is placed on this system and this is depicted by the grey arrows in the model. Stress can cause activation of NADPH oxidases in the apoplast or peroxisomes, which results increases in ROS generation. The sensor/receptor pools also signal significantly enhanced levels of ROS scavenging systems to modulate the ROS levels. In addition, the sensors/receptors mediate a greatly accentuated response such as gene up-regulation to produce proteins such as heat shock proteins which are involved in the amelioration of the stress itself. The component of the system, which involves ROS generation is considered to Stage I in Table I, while the response mechanisms are considered to be Stage II.

can respond to different levels of stress exposure, with a mild exposure generally enhancing the capacity of the cell to resist further or future exposure to that particular stress, or to another stress. This understanding can be used in a practical manner as demonstrated by the use of mild stress treatments to enhance resistance to other stresses and particularly good examples of this are found in the literature on chilling injury. In that literature, mild heat treatment(Lurie, 1998), temperature preconditioning using intermediate temperatures (Wang et al., 1992) and a combination of the two (Wang, 1994) have been shown to increase resistance to subsequent exposures to chilling temperatures. Other examples of the use of mild stress treatments to enhance stress tolerance in postharvest systems are discussed by Toivonen (2003b).

\section{CELLULAR LOCALIZATION OF SYSTEMS FOR SCAVENGING REACTIVE OXYGEN SPECIES}

The complexity and diversity of oxygen radical scavenging systems is parallel to the complexity of their sites of production (Fig. 3). Enzymatic antioxidants work in tandem to detoxify reactive oxygen species. For example, superoxide dismutase converts the superoxide anion to hydrogen peroxide (Mittler, 2002). Hydrogen peroxide must also be scavenged in order to avoid both its direct oxidizing capacity and its propensity to react with superoxide anion in the presence of a divalent iron ion to form the highly reactive hydroxyl radical (Schopfer et al., 2001). Detoxification of hydrogen peroxide can be accomplished with peroxidase, ascorbate peroxidase, glutathione peroxidase, thioredoxin peroxidase, and/or catalase (Mittler, 2002). If the enzyme is ascorbate peroxidase, ascorbate acts as a scavenger of peroxide and the oxidized ascorbate is subsequently recycled via the action of monodehydroascorbate reductase (Mittler, 2002). Ascorbate and glutathione can operate as secondary components of scavenging systems to detoxify lipid hydroperoxides when they are formed, and if conditions are right, the oxidized form of these scavengers can be recycled back to their reduced forms in the process (Shewfelt and Purvis, 1995). They can also work in concert to regenerate ascorbate from dehydroascorbate (Mittler, 2002). Glutathione peroxidase and glutathione reductase can also operate as a fully regenerating detoxification process (Mittler, 2002). Tocopherol and carotenoids function as direct scavengers of lipophilic reactive oxygen species, most often lipid hydroperoxides; however they are coupled to the ascorbate-glutathione system mentioned above (Shewfelt and Purvis, 1995). Alternate oxidase provides an alternate electron flow, which can reduce the production of reactive oxygen species in damaged or inhibited electron transport chains (Mittler, 2002). However, when the production of reactive oxygen species exceeds the capacity of these self-regenerating systems to cope, a decline in many or all of the antioxidants may occur. The most highly profiled component in this regard is ascorbate, which is often used to assess nutritional changes as well as oxidative stress in postharvest studies (Toivonen, 2002a).

It is clear that the protection systems in plant cells is quite complex, and in many cases the components are tightly linked and there appears to be a great deal of redundancy in the systems involved in scavenging reactive oxygen species (Mittler, 2002). Linkage and redundancy provide for a robust and flexible system with great ability to regenerate itself. Therefore, it becomes obvious that the development of oxidative injury in postharvest situations is indicative that significant stress is indeed being applied to the particular tissue in question. This brings a question as to how well the many fruit and vegetables are adapted to apparently gentle postharvest handling procedures. Are these procedures, in fact, rather harsh? That question can be addressed by examining the information available in the literature.

\section{OXIDATIVE STRESS OBSERVATIONS IN THE POSTHARVEST LITERATURE}

While there have been many reports of oxidative damage in fruit and vegetables (see Hodges et al., 2004), the stress where there has been the most detailed mechanistic studies has been with chilling injury (Purvis, 2004). There are several points that stand out and will be discussed here.

Most postharvest procedures can lead to increases in oxidative stress and/or injury, and the latter is confirmed by increases in membrane leakage, except the case of low oxygen atmospheres. Low oxygen seems to be the one exception where most reports indicate a reduction or control of oxidative injury. The reason for this may be that low oxygen actually slows oxidative electron transport and minimizes other 

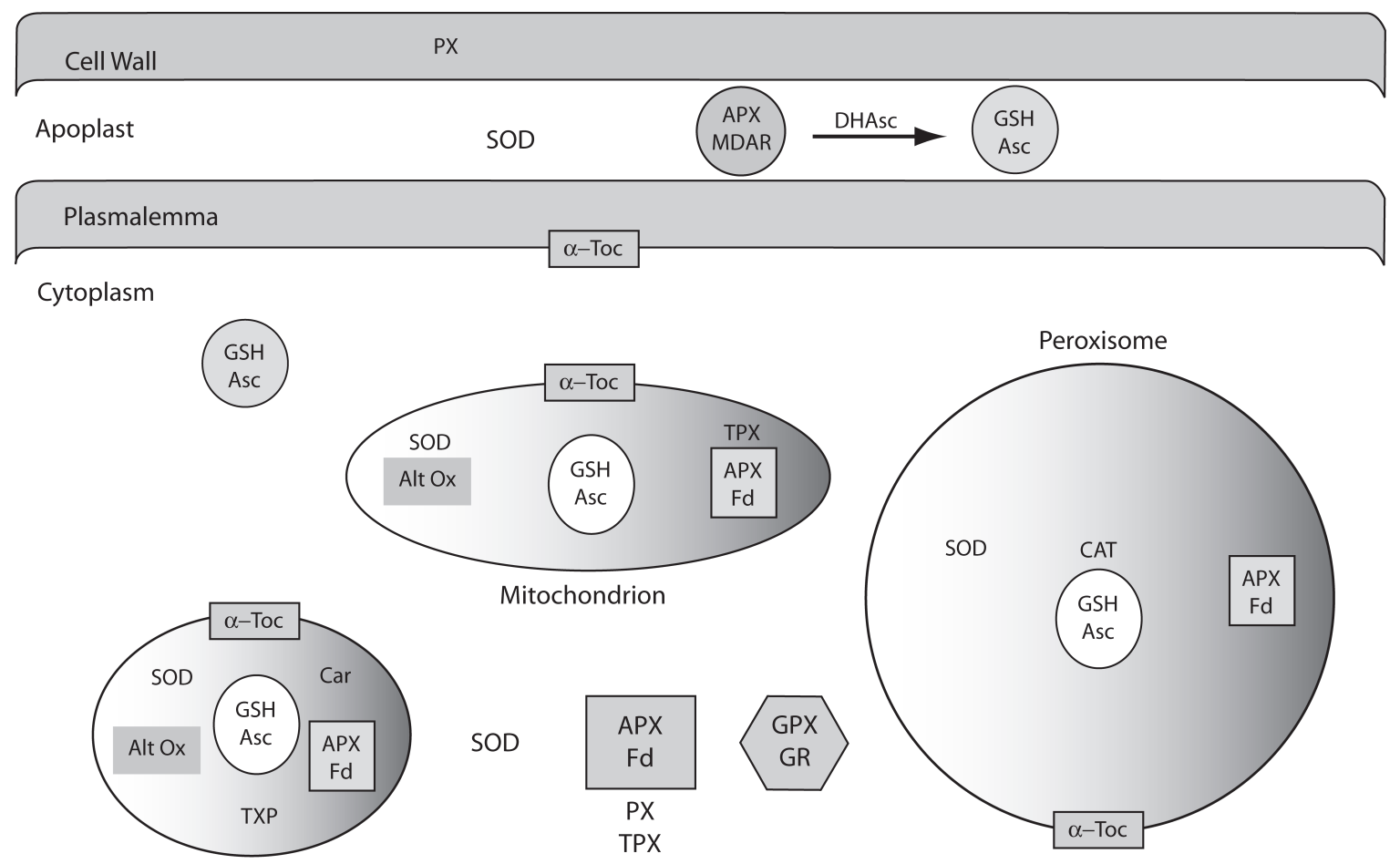

Chloroplast

oxidative processes in the cell (Kader, 1985). While the benefits of low oxygen cannot be argued, it must be remembered that if oxygen levels are brought to extremely low levels, anoxic conditions can develop. In such cases, oxidative injury is only realized when the tissue is returned to higher oxygen levels (Monk et al., 1987). Such a case has been reported with bagged salads which can be sealed in packages where the oxygen levels often drop below 0.5\% (Smyth et al., 1998). The lettuce does not show browning while in the intact package, but shows accelerated browning once the package is opened to the normal atmosphere (Smyth et al, 1998).

While there appear to be many different manifestations of visible injuries that are associated with oxidative stress, they all share similar features, i.e., the measured biochemical oxidative stress indicators are common for most oxidative stresses induced by the different postharvest procedures (Hodges, 2003). This supports the concept that most oxidative injuries have similar mechanisms by which response to the stress is achieved and for the events that lead to injury (Lesham and Kuiper, 1996; Neill et al., 2002). However, there is some deviation from this in that some components of the antioxidant protection system (i.e., nonenzymatic antioxidants and antioxidant enzymes) may not respond in the same manner in all cases of oxidative stress. This divergence is likely associated with at least two factors. 1) The complexity of the reactive oxygen species generating sites in the plant tissues and the possibility that a particular site may be more affected than others by a particular stress (see discussion regarding Fig. 1). 2) The level and duration of stress applied at the time of sampling in each particular case (see discussion regarding Table 1).

There are many unanswered questions left by the current literature in regards to different aspects of oxidative stress for many of the postharvest procedures that have been identified to be associated with oxidative stress (Hodges et al., 2004). This underlies the need for an increased depth of research on oxidative stress in postharvest systems (Toivonen, 2003 a, 2003b).

\section{POTENTIAL CAUSES OF POOR UNDERSTANDING IN POSTHARVEST STUDIES OF OXIDATIVE STRESS}

A common approach to measuring stress in postharvest situations is to sample at a particular point in time of exposure to a treatment or a particular set of storage conditions. However, oxidative homeostasis requires a balance of reactive oxidation species production and scavenging
Fig. 3. Reactive oxygen species scavenging systems in plant cells and the sites at which they are located. Diagram is based on information from Mettler (2002). Abbreviations: $\mathrm{PX}=$ peroxidase, $\mathrm{SOD}=$ superoxide dismutase, $\mathrm{APX}$ $=$ ascorbate peroxidase, $\mathrm{MDAR}=$ monodehyroascorbate reductase, $\mathrm{DHAsc}$ $=$ dehydroascorbate, $\mathrm{GSH}=$ reduced glutathione, $\mathrm{Asc}=$ ascorbate,$\alpha$-toc $=$ $\alpha$-tocopherol, Alt $\mathrm{Ox}=$ alternative oxidase, $\mathrm{TPX}=$ thioredoxin peroxidase, $\mathrm{Car}=$ carotenoids, $\mathrm{Fd}=$ ferrodoxin, $\mathrm{GPX}=$ glutathione peroxidase, and $\mathrm{GR}$ $=$ glutathione reductase

capacity (Mittler, 2002). The maintenance of a homeostatic condition implies that there are a considerable number of dynamic processes always ongoing as represented in Figs. 1-3. Since oxidative balance is a dynamic process, studies should reflect this and have multiple time samplings designed into the experiments. As an example, some studies on internal browning of pears have involved simple point-in-time experiments (Lentheric et al., 1999), which results in limited amount information regarding the onset and development of the disorder. A case in point as to how such data can be limiting is demonstrated in a study where water soluble antioxidant content of 'Delicious' was found to be twice that of 'Empire' in Ontario, Canada (MacLean et al., 2003). From this one might interpret that 'Delicious' should be more resistant to internal browning disorders than 'Empire'. In fact, the opposite is true, 'Empire' is found to be more resistant to internal browning disorders than 'Delicious' in Ontario (J. DeEll, Ontario Ministry of Agriculture, personal communication, 2003). At the other end of the spectrum, time course experiments in pears have brought a great deal of understanding with regard to the processes leading to development of internal browning of pears (Veltman et al., 2003). In the case of 'Delicious' and 'Empire' apples in Ontario, a time course analysis revealed that water soluble antioxidants were very stable throughout storage for 'Empire' but declined significantly for 'Delicious' (MacLean et al., 2003). This second dynamic analysis provided a set of data that could explain the differences in susceptibility to internal browning disorders that have been observed. This dynamic analysis not only allows one to understand the response to storage stress of susceptible and resistant cultivars, but also demonstrates that the absolute level of antioxidants within the tissues may not be as important as whether they can be maintained by the antioxidant protection system when the tissue is subjected to stressful (i.e., commercial storage) conditions.

As stated above, the process of controlling oxidative damage in plant tissues is dynamic, matching scavenging capacity with reactive oxygen species production. Generally, superoxide anion and, subsequently, hydrogen peroxide are the primary reactive oxygen species produced inside 
and outside of the cell (Fig. 1). One of the challenges the plant cell faces is that it must be able to detoxify the reactive species being produced. This is particularly the case for hydrogen peroxide which is generally produced as a dismutation product of the superoxide anion. Therefore the hydrogen peroxide detoxification capacity (e.g., peroxidase activity) must be matched with the detoxification capacity for superoxide anion (e.g., superoxide dismutase activity), otherwise there will be a high potential for accumulation of the highly reactive hydroxyl radical (Mittler, 2002, Stahl and Sies, 2002). Work in regards to comparing cultivars of apples that are either highly susceptible to cut-edge browning with those that are resistant has shown that a strong relationship is found between the ratio of superoxide dismutase to peroxidase activity and the browning potential of that cultivar (Table 2). In contrast, measurements of POD and SOD activities themselves were not found to be associated with cutedge browning. These results demonstrate the importance of measuring as many of the components of the antioxidant protection system as is possible. This allows the interpretation of how these components relate to each other and how their relation translates to control or lack of control of reactive oxygen species accumulation in the tissues.

Another issue is that oxidative stress can be experienced differentially in different tissue types within the same fruit or vegetable. One example of this is that the susceptibility of apples to develop internal browning during storage increases with maturity at harvest (Toivonen, 2003a). This is associated with changes in antioxidant systems in the fruit flesh. In contrast, antioxidant systems in peel tissues increase with maturation of the fruit and this is associated with a reduction in susceptibility to scald (Toivonen, 2003a). These observations highlight the issues facing those working at solving fruit storage disorders, in that susceptibility to one type of oxidative disorder may be quite different than another type in the same fruit. The differential physiology of flesh and peel must be better understood if improvements in handling procedure of tree fruits destined for storage are to be achieved. There is at least one report, which has demonstrated differential physiology between peel and flesh in apples (Lara and Vendrell, 2003). Hopefully more work of this type will be initiated in the future.

One issue that most researchers must contend with on an ongoing basis is that of potential for artifacts in the experiment protocol. Since the study of oxidative stress in postharvest situations is relatively new, procedures, materials and protocols have yet to be adequately developed. It may be advisable that researchers take time to view literature in the plant ecophysiology or stress physiology forums or even medical forums to develop more background knowledge in regards to methodology since those fields have long experience in oxidative stress research. One example of a potential artifact that one may run into is the possible function of a reagent as a reactive oxygen species scavenger. Benzoate, adenine, mannitol, formate, and thiourea are all scavengers of the highly toxic hydroxyl radical (Schopfer et al., 2001). So if any of these reagents are used in buffers or in experimental solutions, the effect of hydroxyl radicals could be minimized or eliminated. If a researcher were unaware of the scavenging function of the reagent, then the results of the experiments might be misinterpreted. Therefore, due diligence is required in design of experiments to evaluate oxidative stress in postharvest systems.

\section{CAN BETTER UNDERSTANDING OF OXIDATIVE STRESS ASSIST IN UNDERSTANDING AND IMPROVING POSTHARVEST PROCEDURES?}

Certainly the extensive work with chilling injury and understanding of the underlying mechanisms has led to approaches to controlling chill- ing injury and advances in scald control in tree fruits have been seen as a result of research activity in that area (Hodges, 2003; Purvis, 2004). These examples demonstrate that basic understanding is often the key to significant advances. One area which has not yet gained appreciable understanding is that of oxidative stress and cut-surface browning in fresh-cut fruit and vegetables. Therefore, a short discussion will be devoted to this area and a new hypothesis presented.

Browning is a phenomenon that has been extensively studied in food science, with the greatest focus of studies being in processed food systems. The understanding developed in processed food systems was then directly transferred more recently to explaining browning and antibrowning treatments in fresh-cut fruit and vegetables. Hence, control of cut-edge browning in fruit and vegetables has been primarily focused on the interaction of polyphenol oxidase with polyphenols on the cut edges, using antioxidant agents in aqueous or coating formulations (Sapers, 1993; Toivonen, 2003b). However, the relationship between browning and polyphenol oxidase and polyphenol content is very poor, in fact it has been suggested that there are no significant correlations when comparing cultivars with different browning susceptibilities (Cantos et al., 2002).

Work in cut lettuce has shown that there is wound reaction and signaling process (i.e., oxidative stress process) involved in the browning phenomenon (Kang and Saltveit, 2003) and that browning occurs beyond those cells which are directly damaged (Ke and Saltveit, 1989). In cut lettuce or any cut product, the cut-damaged surfaces are washed and the polyphenols and polyphenol oxidase in the cut cells would be expected to be, more or less, removed in the process. If browning occurs, it only stands to reason that this would be associated with a wound response and subsequent breakdown of cells below the cut surface (Rolle and Chism, 1987). The cutting process has been demonstrated to initiate a wounding signaling response in lettuce (Kang and Saltveit, 2003). It has been shown that wound signaling in response to abiotic stresses, such as cutting, is mediated through the apoplast of the plant tissues (Pignocchi and Foyer, 2003). The apoplast is a poorly buffered region of the cell and thus ensures that any perturbance caused by biotic or abiotic stresses are not attenuated. A poorly attenuated response ensures that a rapid response to stress is possible. Generally, the primary apoplastic signal molecule is considered to be hydrogen peroxide since it is readily able to permeate membranes and has a moderate half life (Mahalingam and Federoff, 2003). Hydrogen peroxide production is enhanced in a stress situation via induction of increased activity in plasmalemma-bound NADPH oxidases and this is mediated by stressinitiated ion fluxes or $\mathrm{pH}$ changes in the apoplast (Vianello and Macrì, 1991; Felle and Hanstein, 2002). Accumulation of hydrogen peroxide will also lead to enhanced potential for production of hydroxyl radicals which leads to lipid peroxidation and membrane deterioration (Stahl and Sies, 2002).

While the prevailing theory is that antioxidant dips prevent the interaction of polyphenol and polyphenol oxidase (Sapers, 1993; Toivonen, 2003b), an alternate explanation is that anti-browning dip formulations contain ascorbate and other antioxidants that can directly scavenge hydrogen peroxide (Mittler, 2002; Schopfer et al., 2001). Perhaps these dips are active in inhibiting wound signaling and preventing oxidative breakdown of cells below the cut surface, hence the polyphenols cannot come into contact with polyphenol oxidase and initiate the reactions that lead to browning. This theory is supported by the observation that cortical tissue discs from apples do not brown in leakage studies when the discs are suspended in isotonic solutions containing mannitol (Toivonen, personal observation, 2003). Similar effects have been reported in lettuce midrib tissues (Kang and Saltveit, 2003). Mannitol is known to be a hydroxyl radical scavenger (Schopfer etal., 2001), therefore membrane injury and browning may be prevented in this situation by the protective effect mannitol has against lipid peroxidation caused by hydroxyl radical accumulation. Moreover, when discs are mechanically disintegrated, to measure total solute content in the leakage assay, the tissue browning immediately ensues (Toivonen, 
personal observation, 2003). This second observation indicates that the effect is not through inhibition of the polyphenol oxidase reaction, rather it can only be explained as a membrane protection mechanism.

Another characteristic of many anti-browning dips is that their $\mathrm{pH}$ is quite low since they contain large quantities of organic acids and the $\mathrm{pH}$ is partially selected because it has been shown that low $\mathrm{pH}$ will inactivate polyphenol oxidase (Sapers, 1993). However, low apoplastic $\mathrm{pHenhances}$ the activity of many enzymes in the apoplast which are associated with reactive oxygen species production (Vianello and Macrì, 1991) and also some others involved with cell wall softening (Knee, 1982; Almeida and Huber, 1999). It would be expected that there is a lot of membrane injury and this can lead to quality problems which are not always completely resolved. As mentioned above, the low $\mathrm{pH}$ inhibits polyphenol oxidase activity, and so if significant membrane injury occurs, browning reactions would certainly be minimized. In addition, many dips also contain other components which, either knowingly or unknowingly, compensate for the negative effects of the low $\mathrm{pH}$. These other components could include other antioxidants to enhance scavenging capacity and/or calcium salts to stabilize membranes and cell walls against enzyme action.

What if the strategy of a post-cutting treatment was to prevent oxidative injury to the cells in the tissues proximal to the cut edge? There is some preliminary evidence that indicates that an approach which prevents hydroxyl radical accumulation can lead to the prevention of cut-edge browning. Browning in apple discs can be successfully inhibited by an infusion of phosphate buffer adjusted to a $\mathrm{pH}$ of 5 (unpublished data, Toivonen, 2003) which is close to the normal apoplastic $\mathrm{pH}$ of intact plant tissue (Felle and Hanstein, 2002; Pignocchi and Foyer, 2003). At that $\mathrm{pH}, \mathrm{NADPH}$ oxidase activities are minimized and hence woundinduced hydrogen peroxide should be prevented (Stahl and Sies, 2002). As a consequence, potential for hydroxyl radical production should also be minimized. An infusion with a $\mathrm{pH}$ of 5 has been found to result in significantly lower levels of apoplastic hydroxyl radical production as compared to an infusion with a $\mathrm{pH}$ of 3 (unpublished data, Toivonen, 2003). These observations suggest that an approach which is designed to protect the plasmalemma from oxidative stress may indeed lead to an alternative means by which to control cut-edge browning. While these observations are preliminary in nature, they provide an alternate explanation for control of the browning process in cut surfaces of fruit and vegetable tissues. Such a shift in working hypothesis may help researchers in developing improved approaches to controlling prevalent problems that we currently face in managing quality loss in fresh-cut products. By the same token, better understanding of oxidative processes in other postharvest situations should lead to better approaches for prevention of oxidative injury in the future, resulting in novel and improved techniques for maintaining postharvest quality.

\section{SUMMARY}

The foregoing discussion leads to several conclusions in regard to oxidative stress in postharvest systems. First, oxidative stress is a common occurrence and leads to perceptible quality changes in the fruit or vegetable product. Second, research on oxidative stress in tissue response processes in postharvest situations is in its infancy. A greater research effort is required before the knowledge base is such that improvements in quality can be achieved via approaches to reducing or ameliorating oxidative stress. A part of this development in knowledge can be enhanced or accelerated by the use of models such as presented in this discussion. A good example of what can be achieved with modeling is presented by Purvis (2004). And third, an improved understanding of oxidative stress may result in the realization of new paradigms challenging our understanding of postharvest quality degradation. At such time significant advances in control of quality will likely be achieved.

\section{Literature Cited}

Almeida, D.P.F. and D.J. Huber. 1999. Apoplastic $\mathrm{pH}$ and inorganic ion levels in tomato fruit: a potential means for regulation of cell wall metabolism during ripening. Physiol. Plant. 105:506-512.

Cantos, E., J.A. Tudela, M.I. Gil, and J.C. Espìn. 2002. Phenolic compounds and related enzymes are not rate-limiting in browning development of fresh-cut potatoes. J. Agr. Food Chem. 50:3015-3023

del Río, L.A., F.J. Corpas, L.M. Sandalio, J.M. Palmer, M. Gómez, and J.B. Barroso.
2002. Reactive oxygen species, antioxidant systems and nitric oxide in peroxisomes. J. Expt. Bot. 53:1255-1272.

Felle, H.H. and S. Hanstein. 2002. The apoplastic pH of the substomatal cavity of Vicia faba leaves and its regulation responding to different stress factors. J. Expt. Bot. 53:73-82.

Hodges, D.M. (ed.). 2003. Postharvest oxidative stress in horticultural crops. Food Products Press, New York.

Hodges, D.M., G.E. Lester, K.D. Munro, and P.M.A. Toivonen. 2004. Oxidative stress: Importance for postharvest quality. HortScience 39:924-929.

Kader, A.A. 1985. An overview of the physiological and biochemical basis of CA effects on fresh horticultural crops, p. 1-9. In: S.M. Blankenship (ed.). Controlled atmospheres for storage and transport of perishable agricultural commodities. N.C. State Univ. Hort. Rpt. 126

Kang, H.-M. and M.E. Saltveit. 2003. Wound-induced increases in phenolic content of fresh-cut lettuce is reduced by a short immersion in aqueous hypertonic solutions. Postharv. Biol. Technol. 29:271-277.

Ke, D. and M.E. Saltveit, Jr. 1989. Wound-induced ethylene production, phenolic metabolism and susceptibility to russet spotting in iceberg lettuce. Physiol. Plant. 76:412-418.

Knee, M. 1982. Fruit softening III. Requirement for oxygen and $\mathrm{pH}$ effects. J. Expt Bot. 33:1263-1269

Lara, I. and M. Vendrell. 2003. Cold-induced ethylene biosynthesis is differentially regulated in peel and pulp tissues of 'Granny Smith' apple fruit. Postharvest Biol. Technol. 29:109-119.

Lentheric, I., E. Pinto, M. Vendrell, and C. Larrigaudiere. 1999. Harvest date affects the antioxidative systems in pear fruits. J. Hort. Sci. Biotechnol. 74:791-795.

Lesham, Y.Y. and P.J.C. Kuiper. 1996. Is there a GAS (general adaptation syndrome) response to various types of environmental stress? Biol. Plant. 38:1-18.

Lurie, S. 1998. Postharvest heat treatments. Postharv. Biol. Technol. 14:257-269.

MacLean, D.D., D.P. Murr, and J.R. DeEll. 2003. A modified total oxyradical scavenging capacity assay for antioxidants in plant tissues. Postharv. Biol. Technol. 29:183-194.

Mahalingam, R. and N. Federoff. 2003. Stress response, cell death and signalling: the many faces of reactive oxygen species. Physiol. Plant. 119:56-68.

Mittler, R. 2002. Oxidative stress, antioxidants and stress tolerance. Trends Plant Sci. 7:405-410.

Monk, L.S., R. Braendle, and R.M.M Crawford. 1987. Catalase activity and post-anoxic injury in monocotyledonous species. J. Expt. Bot. 38:233-246.

Neill, S.J., R. Desikan, A. Clarke, R.D. Hurst, and J.T. Hancock. 2002. Hydrogen peroxide and nitric oxide as signaling molecules in plants. J. Expt. Bot. 53:1237-1247.

Overmyer, K., M. Broshé, and J. Kangasjärvi. 2003. Reactive oxygen species and hormonal control of cell death. Trends Plant Sci. 8:335-342.

Pignocchi, C., and C.H. Foyer. 2003. Apoplastic ascorbate metabolism and its role in the regulation of cell signaling. Curr. Opin. Plant Biol. 6: 370-389.

Purvis, A.C. 2004. Regulation of oxidative stress in horticultural crops. HortScience 39:930-932.

Rolle, R.S. and G.W. Chism. 1987. Physiological consequences of minimally processed fruits and vegetables. J. Food Qual. 10:157-177.

Sapers, G.M. 1993. Browning of foods: Control by sulfites, antioxidants and other means. Food Technol. 47(10):75-84

Scandalios, J.G. 1993. Oxygen stress and superoxide dismutases. Plant Physiol 101:7-12.

Schopfer, P., C. Plachy, and G. Frahry. 2001. Release of reactive oxygen intermediates (superoxide radicals, hydrogen peroxide, and hydroxyl radicals) and peroxidase in germinating radish seeds controlled by light, gibberellin, and abscisic acid. Plant Physiol. 125:1591-1602.

Shewfelt, R.L. and A.C. Purvis. 1995. Toward and comprehensive model for lipid peroxidation in plant tissue disorders. HortScience 30:213-218.

Smyth, A.B., J. Song, and A.C. Cameron. 1998. Modified atmosphere packaged cut iceberg lettuce: Effect of temperature and $\mathrm{O}_{2}$ partial pressure on respiration and quality. J. Agr. Food Chem. 46:4556-4562.

Stahl, W. and H. Sies. 2002. Introduction: Reactive oxygen species, p. 2. In: Research monographs: Reactive oxygen species. Institut $\mathrm{fr}$ Physiologische Chemie I, Heinrich-Heine-Universitat-D sseldorf. Accessed on 29 Sept. 2003. http://www. uni-dusseldorf.de/WWW/MedFak/PhysioChem/index.html.

Toivonen, P.M.A. 2003a. Effects of storage conditions and postharvest procedures on oxidative stress in fruits and vegetables, p. 69-90. In: D.M. Hodges (ed.). Postharvest oxidative stress in horticultural crops. Food Products Press, New York.

Toivonen, P.M.A. 2003b. Postharvest treatments to control oxidative stress in fruits and vegetables, p. 225-246. In: D.M. Hodges (ed.). Postharvest oxidative stress in horticultural crops. Food Products Press, New York.

Veltman, R.H., I. Lentheric, L.H.W. Van der Plas, and H.W. Peppelenbos. 2003. Internal browning in pear fruit (Pyris communis L. cv. Conference) may be a result of a limited availability of energy and antioxidants. Postharvest Biol. Technol. 28:295-302.

Vianello, A. and F. Macrì. 1991. Generation of superoxide anion and hydrogen peroxide at the surface of plant cells. J. Bioenerg. Biomembr. 23:409-423.

Wang, C.Y. 1994. Combined treatment of heat shock and low temperature conditioning reduces chilling injury in zucchini squash. Postharvest Biol. Technol. 4:65-73.

Wang, C.Y., G.F. Kramer, B.D. Whitaker, and W.R. Lusby. 1992. Temperature conditioning increases tolerance to chilling injury and alters lipid composition in zucchini squash. J. Plant Physiol. 140:229-235. 